\title{
Simultaneous predictive value of NT-proBNP and CA-125 in patients newly diagnosed with advanced heart failure: preliminary results
}

Andrzej Folga ${ }^{1}$, Krzysztof J. Filipiak², Artur Mamcarz ${ }^{1}$, Elzbieta Obrebska-Tabaczka ${ }^{3}$, Grzegorz Opolski

\author{
${ }^{13}$ rd Department of Internal Medicine and Cardiology, Medical University of Warsaw, \\ Poland \\ 21st Department of Cardiology, Medical University of Warsaw, Poland \\ ${ }^{3}$ Cardiology Department, Hospital of Dzialdowo, Poland
}

Submitted: 29 November 2011

Accepted: 30 November 2011

Arch Med Sci 2012; 8, 4: 637-643

DOI: $10.5114 /$ aoms.2012.30287

Copyright $\odot 2012$ Termedia \& Banach

\author{
Corresponding author: \\ Andrzej Folga MD, PhD \\ $3^{\text {rd }}$ Department of Internal \\ Medicine and Cardiology \\ Medical University of Warsaw \\ Szpital Solec \\ 93 Solec \\ 00-382 Warsaw, Poland \\ Phone: +4822 2506253 \\ E-mail: and.folga@gmail.com
}

\begin{abstract}
Introduction: New markers of cardiac events and new monitoring methods which can improve care of patients with advanced heart failure (HF) are still being looked for.

Material and methods: Sixty-five patients below 75 years old (mean age: 60.34 \pm 9.54 years), hospitalized with the first manifestation of HF (left ventricular ejection fraction $\leq 40 \%$ ) and New York Heart Association (NYHA) class II-IV symptoms, not optimally treated before the study, were included. Blood samples for NT-proBNP and CA-125 were taken at baseline and during the 12-month followup period. The doses of $\beta$-adrenolytics and angiotensin-converting enzyme (ACE) inhibitors were titrated to maximal tolerated ones according to the guidelines in 1-year follow-up. The endpoint was established as overall death and time to death.

Results: Worse prognosis was observed in groups with: 1) NT-proBNP and CA-125 above medians (OR $=492.9, p=0.006), 2$ ) baseline higher NT-proBNP and $\mathrm{CA}-125$ (HR $=0.016, p<0.001), 3)$ increased or stable marker levels during the first 3 months after treatment implementation.

Conclusions: Elevated values of NT-proBNP and CA-125 are found as the independent death risk factors. The group with initial elevated NT-proBNP and CA-125 concentrations had a worse prognosis. Changes in NT-proBNP and CA-125 levels after treatment implementation predict unfavourable cardiovascular events with better CA-125 than NT-proBNP performance.
\end{abstract}

Key words: heart failure, mortality, NT-proBNP, CA-125, prognosis.

\section{Introduction}

Chronic heart failure (HF) continues to be a disease with high annual mortality. Despite advances in the development of new treatment and diagnostic methods and the introduction of medication reducing the mortality rate and the occurrence of unfavourable cardiovascular events, there exists a need for finding new methods of risk stratification and identification of the group of patients at the highest risk of occurrence of unfavourable cardiovascular events. Neurohormonal activation is an additional factor, apart from classic echocardiographic and clinical parameters [1], facilitating the stratification of HF patients. B-type natriuretic peptide 
(BNP) is one of the markers of neurohomonal activation. Its position as both a diagnostic and prognostic HF biochemical marker is already well established. There is a paucity of data on the predictive value of another marker accompanying HF CA-125. There are no detailed comparisons of its predictive value with BNP, in particular in the population of people with newly diagnosed HF, which has been improperly treated so far.

The aim of the study was to carry out a prospective evaluation of predicative value of simultaneous determination of CA-125 and NT-proBNP levels in a group of patients suffering from newly diagnosed HF and who had not been administered $\beta$-blockers (BB) and/or angiotensin-converting enzyme inhibitors (ACEI) so were treated not according to the guidelines before inclusion in the study. We were interested to observe how the biochemical marker concentrations (NT-proBNP and CA-125) would change in the population of patients with advanced heart failure not optimally treated when they received proper treatment.

Table I. Demographic characteristics at baseline

\begin{tabular}{|c|c|}
\hline Age & $60.34 \pm 9.54(31-75)$ \\
\hline Sex (male) & $47(72.3 \%)$ \\
\hline Body mass index $\left[\mathrm{kg} / \mathrm{m}^{2}\right]$ & $28.25 \pm 6.31[18.8-58.7]$ \\
\hline Previous myocardial infarction & $24(36.9 \%)$ \\
\hline Hypertension & $29(44.6 \%)$ \\
\hline Diabetes mellitus & $12(18.5 \%)$ \\
\hline Chronic obstructive pulmonary & y disease $9(13.9 \%)$ \\
\hline Renal failure & $51(78.5 \%)$ \\
\hline Pacing & $3(4.6 \%)$ \\
\hline Smoking & $26(40 \%)$ \\
\hline Previous CABG/PCI & $2(3.1 \%)$ \\
\hline $\begin{array}{c}\text { NYHA class } \\
\cdot \text { II } \\
\cdot \text { III } \\
\cdot \text { IV }\end{array}$ & $\begin{array}{c}13(20 \%) \\
36(55.4 \%) \\
16(24.6 \%)\end{array}$ \\
\hline EF LV [\%] & $30.15 \pm 6.52[15.5-40]$ \\
\hline LVM index $\left[\mathrm{g} / \mathrm{m}^{2}\right]$ & $178.85 \pm 45.1[105.05-346.29]$ \\
\hline Atrial fibrillation & $19(29.2 \%)$ \\
\hline \multicolumn{2}{|l|}{ Treatment before study } \\
\hline$\beta$-Blockers & $11(16.9 \%)$ \\
\hline ACE inhibitors & $15(23.1 \%)$ \\
\hline Spironolactone & $9(13.8 \%)$ \\
\hline Diuretics & $25(38.5 \%)$ \\
\hline Digitalis & $15(23.1 \%)$ \\
\hline Statins & $15(23.1 \%)$ \\
\hline Anticoagulants & $13(20 \%)$ \\
\hline Aspirin & $16(24.6 \%)$ \\
\hline
\end{tabular}

\section{Material and methods}

Sixty-five patients below 75 years of age (mean age: 60.3 years), who were hospitalised with symptoms of HF, participated in the study. The inclusion criteria for the study were the following: newly diagnosed HF, NYHA class II-IV, left ventricular ejection fraction $(E F) \leq 40 \%$, age below 75 years, patients untreated with BB and/or ACEl over the last 6 months before their inclusion in the study despite medical indications, the patient's written consent to participate in the study. The exclusion criteria were the following: failure to meet the inclusion criteria for the study and, additionally, prior myocardial infarct in the past six months, haemodynamically significant heart defect requiring cardiosurgical intervention, intravenous administration of inotropic agents during hospitalisation, occurrence of other serious diseases outside the cardiovascular system with a bad prognosis. The study participants were selected from patients hospitalized in the Hospital of Dzialdowo admitted with symptoms of HF and complied with conditions of inclusion and exclusion criteria.

The study was approved by the local bioethical commission at the Medical University of Warsaw. All patients consented to participate in the study. Baseline characteristics of the experimental group are presented in Table I.

After the patients were included in the study, medical examinations were performed, and serum samples were collected to determine basic biochemical parameters and NT-proBNP and CA-125 concentrations. Chest X-rays, electrocardiograms, and echocardiograms were performed. Depending on the patient's clinical condition, HF therapy was implemented according to the guidelines of the European Society of Cardiology with all of the patients being put on ACEI and BB therapy. The patients were followed ambulatorily in the $3^{\text {rd }}, 6^{\text {th }}$ and $12^{\text {th }}$ months, during which they underwent medical examination, had an echocardiogram performed and their pharmacological treatment was modified. Our aim was to achieve the maximum recommended dosage of $\mathrm{BB}$ and $\mathrm{ACEl}$ during the observation period. The 1-year-long observation of the last patient participating in the study was completed at the end of 2004. No patients were lost during the observation period.

Upon the patient's admission to hospital, blood samples were collected in order to obtain results of basic biochemical tests. Serum samples were collected to determine NT-proBNP (Roche Diagnostics Ltd. ${ }^{\circledR}$, Poland) and CA-125 (Siemens Healthcare Diagnostics Inc. ${ }^{\circledR}$, USA) concentrations. The serum was frozen at a temperature of $-30^{\circ} \mathrm{C}$ and next at $-80^{\circ} \mathrm{C}$ until the determination was performed. In the subsequent months of the observation period, biochemical tests were performed depending on 
the patient's clinical condition and therapeutic needs.

The endpoints of the study were the patient's death and the period until death occurred.

\section{Statistical analysis}

The influence of concentrations of the markers NT-proBNP and CA-125 as well as echocardiographic, demographic and biochemical parameters at endpoints were analysed by means of the multifactorial logistic model and the proportional hazard models for the death and the time of death occurrence, respectively. Parameters of continuous nature were classified by adopting the median as the cut-off point.

The goodness-of-fit of logistic models was verified using the Hosmer-Lemeshow model. Goodnessof-fit of proportionate hazard models was verified using Schoenfeld residuals. The relevant calculations were performed with the STATA statistical software, version 8.2.

\section{Results}

In cases of death, both due to cardiovascular and extracardiac reasons, clinical data were obtained from the family or the general practitioners in the case of death outside hospital or from medical documentation if the patient died in hospital.

During the 1-year-long period, altogether 11 patients died. All cases of death were caused by cardiovascular factors - decompensation of HF (annual total mortality of 16.9\%). Aetiology of heart failure was: coronary artery disease $52.4 \%$, hypertension $44.6 \%$, dilated cardiomyopathy $1.5 \%$, myocarditis $1.5 \%$.

Patients with elevated values of plasma concentrations of NT-proBNP and CA-125, compared to the group with values below the median, had a worse prognosis ( $\mathrm{OR}=492.9$ [5.8, 42000]; $95 \% \mathrm{Cl}$, $p=0.006$ ). In addition, patients with elevated values of one of the markers, compared to the group with the NT-proBNP and CA-125 values below the median, showed a tendency for more frequent occurrence of cardiovascular death. However, the differences were not statistically significant $(\mathrm{OR}=19.7[0.63,622.5] ; 95 \% \mathrm{Cl}, p=0.09)$.

The median of the NT-proBNP concentrations determined on the day of inclusion in the study was $1754.0 \mathrm{pg} / \mathrm{ml}$. The median value for the CA-125 antigen on the day of inclusion in the study was 24.19 $\mathrm{IU} / \mathrm{ml}$. The average marker values were: NT-proBNP $=14184.87 \mathrm{pg} / \mathrm{ml}$ and $\mathrm{CA}-125=66.01 \mathrm{IU} / \mathrm{ml}$.

Table II. Results of multifactorial analysis of all parameters. Analysed parameters and the occurrence of cardiovascular death (total mortality*), final model

\begin{tabular}{|lcc|}
\hline$N=65$ & OR $[95 \% \mathrm{Cl}]$ & Value of $p$ \\
\hline NT-proBNP and CA-125 & & \\
\hline $1>$ median vs. both < median & $19.7[0.63,622.5]$ & 0.09 \\
\hline Both > median vs. both < median & $492.9[5.8,42000]$ & 0.006 \\
\hline Delta CA-125 > median & $17.9[1.3,250.9]$ & 0.032 \\
\hline
\end{tabular}

Goodness-of-fit: $p=0.33$. *all deaths were caused by cardiovascular factors
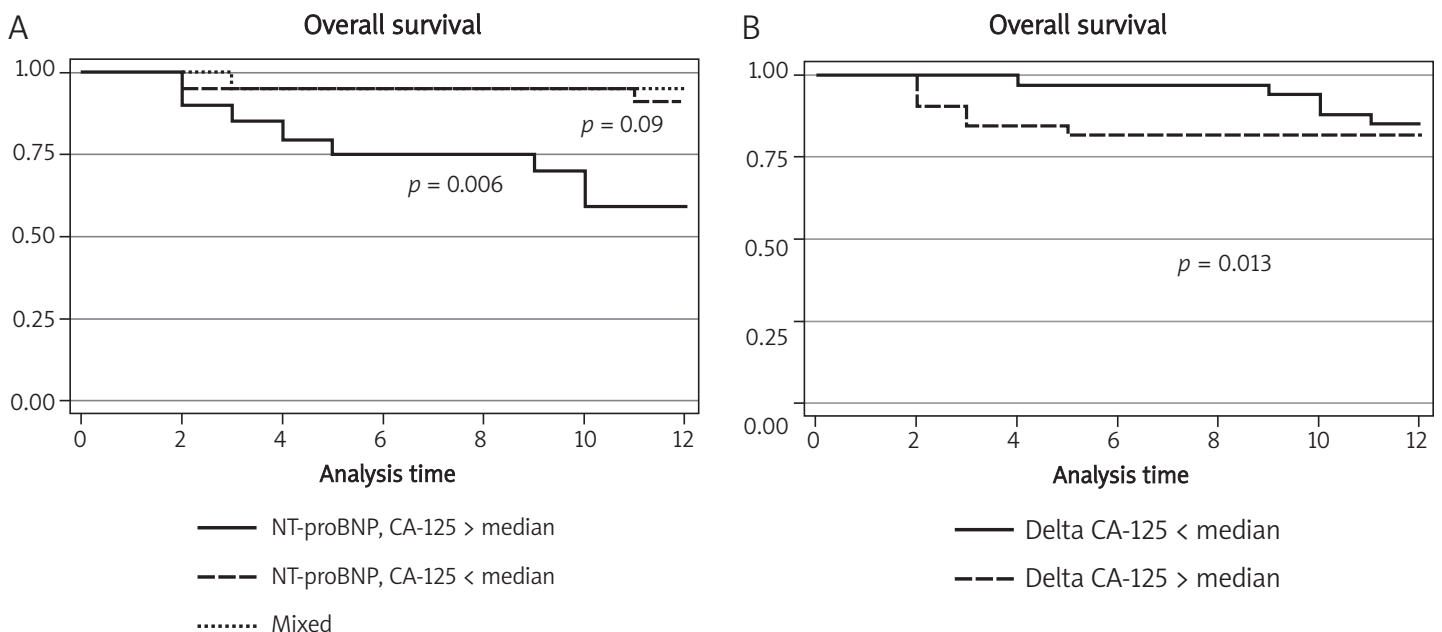

Figure 1. Total mortality in the groups depending on the NT-proBNP and CA-125 values above or below the median at baseline and CA-125 changes during the first 3 months 
Table III. Median values for the initial concentrations of NT-proBNP and CA-125, and for delta CA-125 and delta NT-proBNP

\begin{tabular}{|lc|}
\hline Parameters & Median \\
\hline CA-125 [U/ml] & 24.19 \\
\hline Delta CA125 (measured in 3rd month - base measurement) & -1.79 \\
\hline NT-proBNP [pg/ml] & 1754 \\
\hline Delta NT-proBNP (measured in the 3rd month - baseline measurement) & -71 \\
\hline Goodness-of-fit: $p=0.33 .{ }^{*}$ all deaths were caused by cardiovascular factors &
\end{tabular}

Table IV. The influence of analysed parameters on the time of death, final model

\begin{tabular}{|lcc|}
\hline$N=65$ (11 deaths) & HR $[95 \% \mathrm{Cl}]$ & Value of $p$ \\
\hline $\begin{array}{l}\text { NT-proBNP and CA-125 } \\
1>\text { median vs. both }>\text { median }\end{array}$ & $0.008[0.01,0.41]$ & 0.003 \\
\hline Both $<$ median vs. both $>$ median & $0.016[0.001,0.16]$ & $<0.001$ \\
\hline Delta CA-125 $>$ median & $7.04[1.50,33.1]$ & 0.013 \\
\hline Delta NT-proBNP $>$ median & $4.0[0.94,16.8]$ & 0.06 \\
\hline
\end{tabular}

Time to death

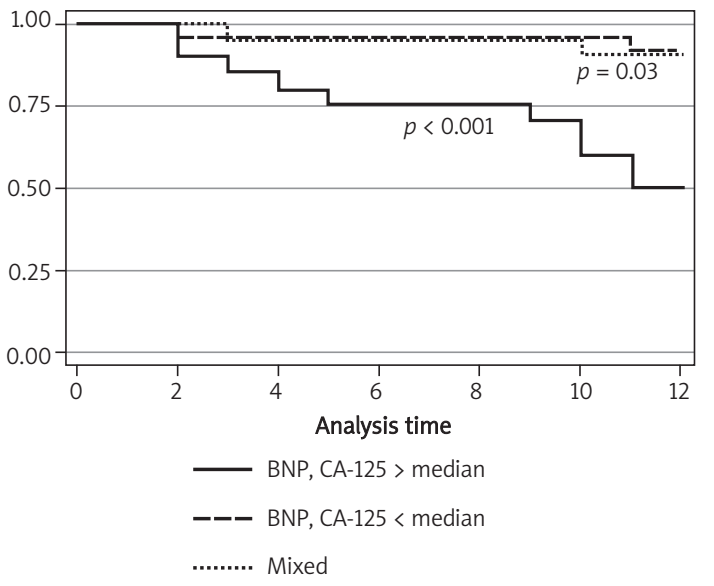

Figure 2. Time to death in the 12-month observation period in 3 groups isolated on the basis of the concentrations of NT-proBNP and CA-125 markers in plasma

The analysis of changes of CA-125 concentrations revealed a statistically significant increase in the risk of death if the plasma concentration of CA-125 was not reduced in the $3^{\text {rd }}$ month of observation compared to the base CA-125 value (the change in CA-125 concentration, called "delta CA-125"), above the median (OR = 17.9 [1.3, 250.9]; $95 \% \mathrm{Cl}, p=0.032$ ). No such dependency was observed for the changes of plasma NT-proBNP in any observation months. The results obtained are presented in Table II and Figure 1.

The median values for initial concentrations of NT-proBNP as well as for CA125 and "delta NT-proBNP" and "delta CA-125" are presented in Table III.

The highest total mortality was noted in the group in which both markers - the initial NT-pro
BNP and the initial CA-125 - were elevated above the median (Figure 1).

\section{Time to death}

Patients from the group with both markers (NTproBNP and CA-125) below the median, compared to the group with both markers above the median, lived longer and this difference was statistically significant $(\mathrm{HR}=0.016$ [0.001, 0.16]; 95\% Cl; $p<0.001)$. The group of patients with values of just one of the markers, NT-proBNP or CA-125, above the median, also had a better prognosis, compared to the group with elevated levels of both markers (Table IV and Figure 2).

In the analysis of changes in plasma concentrations during the first three months of treatment, the lowering of the CA-125 concentration by less than $1.79 \mathrm{U} / \mathrm{ml}(\mathrm{HR}=7.04[1.50,33.1] ; 95 \% \mathrm{Cl}$, $p=0.013)$ was connected with a statistically significant worse prognosis. The lowering of NT-proBNP concentrations during the first three months of treatment by less than $71 \mathrm{pg} / \mathrm{ml}(\mathrm{HR}=4.0[0.94$, 16.8 ]; $95 \% \mathrm{Cl}, p=0.06$ ) revealed a tendency for a worse prognosis.

Changes of NT-proBNP and CA-125 concentrations in plasma during the subsequent months of treatment did not make it possible to assess the risk regarding the time-of-death occurrence. No statistical significance was obtained in the changes of marker concentrations in the subsequent months of observation; however, they remained on the borderline of statistical significance.

A statistically significant degree of correlation was obtained in the study between initial NT-proBNP and initial CA-125 concentration $(R=0.50, p<0.001)$. 


\section{Discussion}

It results from the research which has been conducted so far that NT-proBNP is a strong predictor of the occurrence of death in patients suffering from $\mathrm{HF}[2,3]$, rehospitalisation due to the deterioration of cardiac function, and the occurrence of myocardial infarction in HF patients [2, 4]. It is an independent marker of death occurrence, with a stronger predictive value than left ventricular ejection fraction, plasma creatine concentration, the presence of atrial fibrillation or the NYHA functional class [5]. NT-proBNP is a marker of cardiovascular events not only in a population of patients with $\mathrm{HF}$ but also in a group of patients with aortic aneurysm undergoing vascular surgery. The marker strongly predicts the risk of left ventricular dysfunction after surgery [6].

Few studies have been conducted using the CA-125 antigen as a predictor of death in the course of HF. Its elevated values, however, were connected with an increase in mortality [7, 8]. In the research conducted, both markers proved to be predictors of death in the course of HF. The CA-125 antigen as an ovarian cancer marker exhibits low specificity [9]. Its elevated concentrations occur in different types of cancer and in non-gynaecological diseases [10]. Elevated concentrations of this antigen also occur in HF and are connected with the patients' bad prognosis $[7,8]$. In the course of HF, CA-125 is positively correlated with the NYHA functional class [11, 12], the presence of pleural effusion [13] and pulmonary capillary wedge pressure [14].

In our study, the patients with elevated concentrations of both markers were characterised by over 490-fold increase in death risk in the course of HF, taking into account all parameters assessed in the study. In the analysis of time to death, elevated values of one marker above the median were connected with 92-fold rarer death occurrence, compared to the group of patients with elevated levels of both markers. The group with elevated levels of both markers, compared to the group with marker values below the median, was characterised by nearly 980 -fold higher risk of earlier death.

A single determination of initial concentrations of NT-proBNP and CA-125 markers is the strongest prognostic factor for death in patients suffering from advanced HF.
The average marker values observed in our study for advanced HF are comparable to the results obtained by other authors for NT-proBNP and CA-125 [8, 11]. The maximum value of NT-proBNP (495 $479.0 \mathrm{pg} / \mathrm{ml})$ in one of the patients in our study is probably one of the highest values described in a HF patient. It was determined in a dialysed female patient. The values of subsequent NT-proBNP determinations in this particular patient are presented in Table $\mathrm{V}$. In reports on plasma NTproBNP determination in dialysis patients suffering from renal failure, its significant predictive value for death occurrence is emphasised, despite the presence of renal dysfunction [14, 15]. The possibility of obtaining higher values of NT-proBNP concentrations (even up to 30\%) in samples collected before dialysis treatment, compared to determinations after dialysis, were revealed in other studies [16]. What is important, the results of our study did not change even after excluding this patient.

The maximum single concentration of the CA-125 antigen on the day of the patient's inclusion in the study was $1094 \mathrm{IU} / \mathrm{ml}$. It is a value which was recorded in a female patient suffering from renal failure. No basis was found for diagnosing any type of cancer in additional tests. During a multiple-year observation of this patient, which has not been described in the present study, the patient's clinical condition improved significantly due to the implementation of proper HF treatment. The spectacular course of the CA-125 dynamics in this patient is presented in Table $\mathrm{V}$.

Low specificity of the CA-125 antigen in diagnosing ovarian cancer is emphasised in the literature $-72.0 \%$ [9]. Values $>1000 \mathrm{IU} / \mathrm{ml}$ of CA-125 in plasma increase the specificity of ovarian cancer diagnosis up to $99.1 \%$, but its sensitivity is lowered from $88.6 \%$ to $52.3 \%$ [9]. The values of CA- 125 concentration are elevated in various diseases, such as lung diseases, liver cirrhosis, diseases of the abdominal cavity, and malignant tumours [10].

No reduction in the plasma concentrations of the CA-125 antigen during the first 3 months of treatment, or a reduction by less than $1.76 \mathrm{IU} / \mathrm{ml}$, was connected with an almost 18-fold increase of death risk in the group of patients participating in the study, while changes in the NT-proBNP concentrations $(p>0.1)$ had no influence on death risk. In other studies, changes in CA-125 concentrations

Table V. NT-proBNP and CA-125 concentration in two different patients with the highest NT-proBNP and the highest CA-125 value determined on the day of the patient's inclusion in the study and during follow-up

\begin{tabular}{|lccccc|}
\hline Patient & Parameter & \multicolumn{4}{c|}{ Month of observation } \\
\cline { 3 - 5 } & & 0 & 3 & 6 & 12 \\
\hline No. 1 & NT-proBNP $[\mathrm{pg} / \mathrm{ml}]$ & 495479.0 & 39103.0 & 37638.0 & 4907.0 \\
\hline No. 2 & CA-125 $[\mathrm{IU} / \mathrm{ml}]$ & 1094 & 48.23 & 42.20 & 41.55 \\
\hline
\end{tabular}


were observed in groups of HF patients after the implementation of pharmacological treatment $[7,8,17]$ and after heart transplantation [6]. Patients with elevated CA-125 values or with no reduction of the antigen levels had a worse prognosis - death or deterioration of cardiac function requiring hospitalisation was observed more frequently in this group $[7,8]$.

Time to death in the population of patients under investigation was influenced by the concentrations of NT-proBNP and the CA-125 antigen as well as the change of the CA-125 antigen concentration during the first 3 months of treatment. Changes of NT-proBNP concentrations in plasma were on the borderline of statistical significance $(p=0.06)$.

In a comparison of unfavourable prognostic factors of death and time to death, the NT-proBNP and CA-125 markers remained at the top of the "hierarchy".

Lack of reduction in the concentration of the CA-125 antigen in plasma during the first three months of treatment or a reduction by less than $1.76 \mathrm{IU} / \mathrm{ml}$ was connected with a 7-fold higher increase of shortening the survival time in the group of patients participating in the study. Unfavourable changes of the NT-proBNP concentrations during the first 3 months of treatment showed a tendency for statistical significance of early death.

Unfavourable changes of NT-proBNP concentrations as well as the CA-125 antigen concentrations $[7,8,17]$ were observed in groups of HF patients after the implementation of pharmacological treatment or heart transplantation. Patients with elevated marker values or no reduction in these values had a worse prognosis - the occurrence of death or exacerbation of heart function requiring hospitalisation was observed more frequently in this group of patients $[7,8,17]$. These observations are consistent with the results obtained in our study.

Our study, to the best of our knowledge, is the first one in which a correlation between NT-proB$\mathrm{NP}$ and the CA-125 antigen in the course of HF was revealed together with the better utility of CA-125 changes than NT-proBNP changes for predictive value in the follow-up. An increase in NT-proBNP concentrations is connected with a more advanced pathological process of HF and a bad short-term and long-term prognosis [2,3]. An increase in the CA125 concentrations in the course of HF is also connected with an unfavourable prognosis $[7,8]$. The mutual correlation of both markers indicates a more advanced pathological process in HF. The higher the plasma concentrations of both markers, the worse the patient's prognosis is. The results obtained make it possible to assess the highest unfavourable prognosis for a group of patients with advanced $\mathrm{HF}$ and elevated values of both markers.

The influence of NT-proBNP and CA-125 concentrations on the occurrence of death and time to death was assessed simultaneously in a group of patients with advanced HF, who had been treated in a non-optimal way before. A one-time determination of plasma concentrations of NT-proBNP and CA-125, before the implementation of optimal HF treatment, proved to be the strongest prognostic factor in the assessment of an increased mortality rate in patients in the population under investigation.

The small size of the experimental group and small number of deaths are the limitations of the study. Continuation of the study is required to confirm the results.

In conclusion, on the basis of the preliminary results obtained and the discussion, the following conclusions were formulated with reference to the aims defined at the beginning of the study: Simultaneous elevated initial values of NT-proBNP (> $1754.0 \mathrm{pg} / \mathrm{ml}$ ) and CA-125 (> $24.19 \mathrm{lU} / \mathrm{ml}$ ) are found as the independent death risk factors in the one-year follow-up observation. The group with initial elevated NT-proBNP and CA-125 concentrations had a worse prognosis compared to the group with elevated values of one of the markers. Close observation of changes in NT-proBNP and CA-125 levels during the first 3 months after treatment implementation provides significant guidelines for predicting unfavourable cardiovascular events with better CA-125 than NT-proBNP performance.

\section{References}

1. Mujib M, Desai R, Levitan EB, et al. Prospective population studies of incident heart failure without data on baseline left ventricular ejection fraction. Arch Med Sci 2010; 6: 686-8.

2. Richards M, Troughton RW. NT-proBNP in heart failure: therapy decisions and monitoring. Eur J Heart Fail 2004; 6: 351-4.

3. Masson S, Latini R, Anand IS, et al; Val-HeFT Investigators. Direct comparison of B-type natriuretic peptide (BNP) and amino-terminal proBNP in a large population of patients with chronic and symptomatic heart failure: the Valsartan Heart Failure (Val-HeFT) data. Clin Chem 2006; 52: 1528-38.

4. Perna ER, Macin SM, Cimbaro Canella JP, et al. Importance of early combined $\mathrm{N}$-terminal pro-brain natriuretic peptide and cardiac troponin T measurements for long-term risk stratification of patients with decompensated heart failure. J Heart Lung Transplant 2006; 25: 1230-40.

5. Rienstra M, Van Gelder IC, Van den Berg MP, Boomsma F, Van Veldhuisen DJ. Natriuretic peptides in patients with atrial fibrillation and advanced chronic heart failure: determinants and prognostic value of (NT-)ANP and (NT-pro)BNP. Europace 2006; 8: 482-7.

6. Waliszek M, Waliszek-Iwanicka A, Grycewicz T, et al. Prognostic value of plasma $\mathrm{N}$-terminal pro-B-type 
natriuretic peptide concentration in patients with normal and impaired left ventricular systolic function undergoing surgery for abdominal aortic aneurysm. Arch Med Sci 2011; 7: 642-7.

7. Nagele H, Bahlo M, Klapdor R, Schaeperkoetter D, Rodiger W. CA 125 and its relation to cardiac function. Am Heart J 1999; 137: 1044-9.

8. D'Aloia A, Faggiano P, Aurigemma G, et al. Serum levels of carbohydrate antigen 125 in patients with chronic heart failure: relation to clinical severity, hemodynamic and Doppler echocardiographic abnormalities, and shortterm prognosis. J Am Coll Cardiol 2003; 41: 1805-11.

9. Moss EL, Hollingworth J, Reynolds TM. The role of CA125 in clinical practice. J Clin Pathol 2005; 58: 308-12.

10. Miralles C, Orea M, Espana P, et al. Cancer antigen 125 associated with multiple benign and malignant pathologies. Ann Surg Oncol 2003; 10: 150-4.

11. Kouris NT, Zacharos ID, Kontogianni DD, et al. The significance of CA125 levels in patients with chronic congestive heart failure. Correlation with clinical and echocardiographic parameters. Eur J Heart Fail 2005; 7: 199-203.

12. Varol E, Ozaydin M, Dogan A, Kosar F. Tumour marker levels in patients with chronic heart failure. Eur J Heart Fail 2005; 7: 840-3.

13. Turk HM, Pekdemir H, Buyukberber S, et al. Serum CA 125 levels in patients with chronic heart failure and accompanying pleural fluid. Tumour Biol 2003; 24: 172-5.

14. Austin WJ, Bhalla V, Hernandez-Arce I, et al. Correlation and prognostic utility of B-type natriuretic peptide and its amino-terminal fragment in patients with chronic kidney disease. Am J Clin Pathol 2006; 126: 506-12.

15. Bruch C, Reinecke H, Stypmann J, et al. N-terminal probrain natriuretic peptide, kidney disease and outcome in patients with chronic heart failure. J Heart Lung Transplant 2006; 25: 1135-41.

16. Racek J, Kralova H, Trefil L, Rajdl D, Eiselt J. Brain natriuretic peptide and $\mathrm{N}$-terminal proBNP in chronic haemodialysis patients. Nephron Clin Pract 2006; 103: c162-72.

17. Faggiano P, D’Aloia A, Bignotti T, Dei Cas L. One biologic marker (carbohydrate antigen-CA 125), two different diseases (ovarian cancer and congestive heart failure): practical implications of monitoring CA 125 serum levels. A case report. Ital Heart J 2003; 4: 497-9. 